\title{
Surface Defect Detection Method of Mechanical Parts Based on Target Feature
}

\author{
Chengcheng Wang, Shengqi Guan, Wensen Li, Benben Hong, Hong Liang
}

College of mechanical \& electronic Engineering, Xi'an Polytechnic University, Xi'an, 710048, China

Keywords: Mechanical parts; Surface defect; Target feature; Detection method

\begin{abstract}
Through the analysis of surface defect characteristic about mechanical parts, a new method of based on target feature was presented to surface defect detection of mechanical parts. First of all, the test image is processed by mean-filter in order to eliminate the effect of noise on the detection. Secondly, based on the analysis of the characteristic of the surface defect, the segmentation threshold is set, and segment surface defect of mechanical parts. Finally, the effect of noise and outlier on the detection result was eliminated by morphological filtering. Experiment shows that the method can effectively suppress the image background interference, and more accurately realize the surface defect detection of mechanical parts.
\end{abstract}

\section{Introduction}

In the process of mechanical parts machining and using, the surface of the mechanical parts will produce cracks and other defects. And these defects seriously affect the surface quality of mechanical parts. Therefore, it is important to monitor the surface quality of the machine parts. However, the traditional monitoring method is done by manual off-line sampling. This method depends on the inspection of the personnel, and can not find the defects in real time. Image detection technology can be collected through the image processing, so as to achieve online detection, and image processing based detection technology is the key to image processing algorithm.

At present, for detecting surface defects of mechanical parts based on image processing method, respectively for detecting surface defects of mechanical parts for cutter, strip, gears, bearings and other methods are proposed to detect the spatial domain (edge detection method, zero mean method) and wavelet domain detection algorithm. Among them, zero mean method is constructed with zero mean, and split the defect area by threshold. and this algorithm is simple, but the defective region detection error. Edge detection method is implemented on defect detection by edge defect detection, but this method can only be extracted roughly edge defects, can not detect the complete defect area. The detection algorithm in wavelet domain is decomposed to normal region information and defect area information are separated by wavelet, which can detect the defective area.

However, the segmentation of the defect region depends on the performance of the wavelet filter, and how to construct the wavelet filter is a difficult problem.

In fact, the human visual system under the premise of prior knowledge, always in the field search matching with the prior knowledge of target detection feature, and preferential treatment to these goals, this is a human detection mechanism. If this mechanism is introduced to the mechanical parts surface defect detection using characteristic defects as mechanical parts defect detection conditions, is likely to focus attention to the area where the defect, so as to avoid the influence of environmental factors on the detection, to achieve accurate segmentation of the defect area.To this end, the detection of the target feature set segmentation threshold, to achieve the accurate segmentation of the surface defects of mechanical parts.

\section{Mean filter of surface defect of mechanical parts}

The image collected by industrial field often contains noise, in order to eliminate the impact of the late defect detection, we intend to slide in the collected images mean filtering.

Set the size of the image, and the image is divided into the image window, and then each window 
gray mean:

$$
\begin{aligned}
& F_{I, J}=\frac{1}{9} \sum_{m=0}^{8} \sum_{n=0}^{8} f_{I+M, J+N}, I=\left[\frac{i}{8}\right], J=\left[\frac{j}{8}\right] \\
& 0 \leq i \leq M-1,0 \leq j \leq N-1
\end{aligned}
$$

After the calculation of the value of each sub window gray, the sub window mean instead of window center pixel values; sliding mean filter is realized by a sub window to traverse the whole image.

\section{Surface defect segmentation based on objective analysis}

Under the condition of a priori knowledge, human vision is always concerned with the same characteristics of the field of view and the detection of the target, and the specific characteristics of the area for analysis and processing, in order to improve the detection speed and accuracy. Mechanical parts surface defect sizes and shapes, various kinds of defects; however, these defects and their common characteristics, which is defective region number phase background is always scarce, if can take advantage of this feature can save computer, accurate segmentation of the defect information. Usually rare targets must meet the following conditions:

1) The target area must have a certain degree of division with the background area, and there is a certain degree of contrast;

2) The number of target area is relatively small compared with the number of background area.

In order to meet the above conditions, the formula (1) is as follows:

$$
\left\{\begin{array}{l}
F_{\max }(i, j)>\delta_{\text {otsu }} \\
F_{\text {rarity }(n)}=\operatorname{num}\left[F_{\max }(i, j)\right]_{\delta(n)}-\operatorname{mun}\left[F_{\max }(i, j)\right]_{\delta(n+1)} \\
F_{\text {rarity }(\max )}=\max \left[F_{\text {rarity }(n)}\right]
\end{array}\right.
$$

According to the formula (1), to find out the best rare degree of the corresponding threshold $\delta(n+1)$, using the threshold to break up the region of interest, and the interest area must meet the formula (2):

$$
\left\{\begin{array}{l}
F(i, j) \geq \delta(n+1), \quad F(i, j) \in \text { region }_{(\text {int erest) }} \\
F(i, j)<\delta(n+1), \quad F(i, j) \in \text { region }_{(\text {background) }}
\end{array}\right.
$$

In the example of Figure 1, in third, and fourth times the number of segmentation and detection of region of interest is the maximum difference, therefore, to determine the maximum degree of scarcity; segmentation threshold $\delta(4)$, as shown in Figure 1 (c) shows, can effective defect segmentation information.
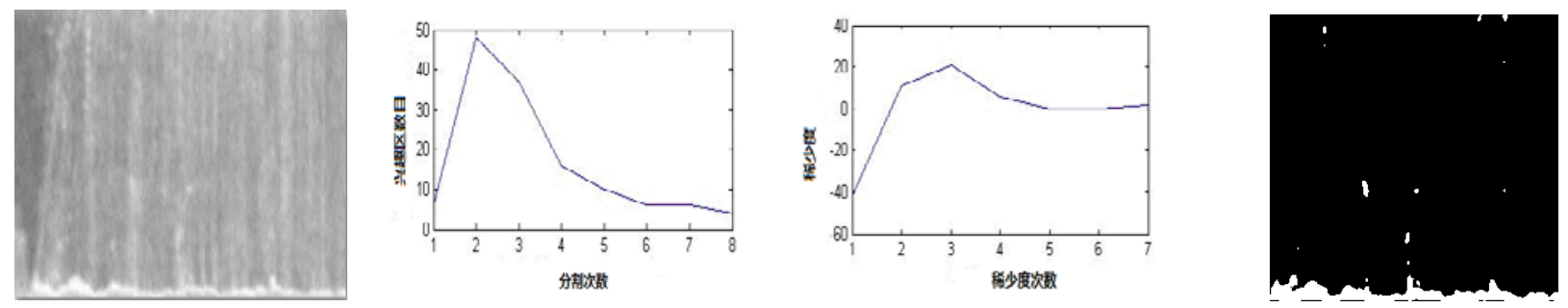

(a)the tool wear image (b)interest area curve $\quad$ (c)sparse degree curve $\quad$ (d)surface defect segmentation results

\section{Morphological filter}

Mechanical parts surface defect image segmentation area, usually contain some noise and small isolated points; in order to eliminate the influence of this part of the information of defects, the first 
expansion, after the corrosion morphology close operation; by closing operation can not only smoothing filter, but also can remove the holes in the region, and to fill up the narrow fracture, slender gully and contour of the gap.

Set the two value image to $\mathrm{I}$, the connected domain is set to $\mathrm{X}$, the structure element is $\mathrm{S}$, and when the origin of a structural element $S$ moves to (x, y), we will make a note of it. At this point, the image $\mathrm{X}$ is closed by the structure element $\mathrm{S}$ computing can be expressed as the formula (3):

$$
\left\{\begin{array}{l}
E=I \Theta S=\left\{x, y \mid S_{x y} \subseteq X\right\} \\
D=E \Theta S=\left\{x, y \mid S_{x y} \cap X \neq \phi\right\}
\end{array}\right.
$$

Figure 1 (c) by morphological filtering, as shown in Figure 2 can effectively filter out noise and smaller isolated points.

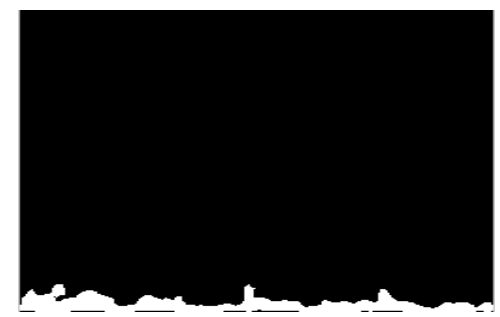

Fig. 2 morphological filtering effect

\section{Experiment and analysis}

In order to verify the effectiveness of this algorithm, we select the cutting tool wear, gear crack and strip defect as the target, and the results are shown in Figure 3. It can be seen that this method can effectively segment the defect region.

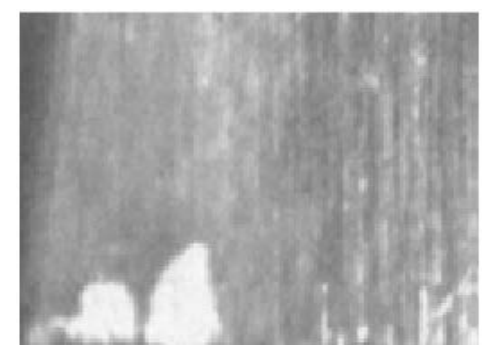

(a)tool wear image

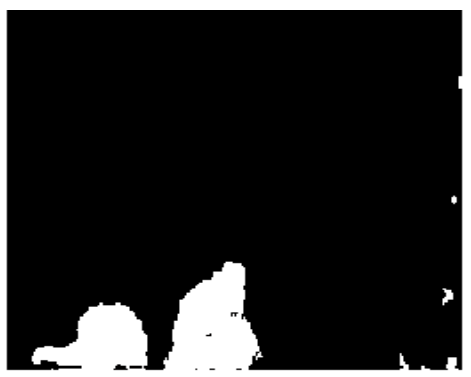

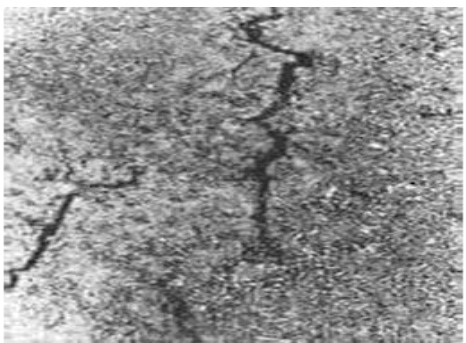

(b)gear crack image

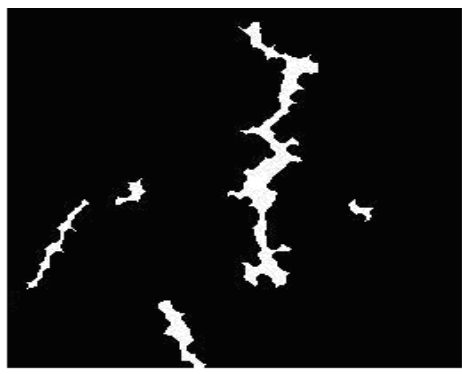

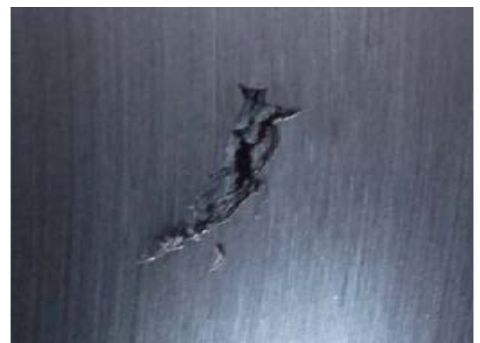

(c)strip defect image



(d)tool wear test results (e)gear crack detection results (f)strip defect detection results

fig. 3 segmentation results of surface defects of mechanical parts

In order to verify the accuracy of the algorithm, we select the 100 pieces of cutting tool wear, gear crack and strip defect image respectively. The specific steps: first, by testing personnel for a test image defects in the region of artificial markers, and calculate the defect area; then, the test image by the algorithm of segmentation, and statistics of the foreground area. Finally, the artificial markers area and the segmentation of defect area were compared, when the error is less than $10 \%$, that be detected accurately. Test results show that the three kinds of defect detection accuracy rate reached 98\%, 95\%, 97\%, with a higher detection accuracy. 


\section{Conclusions}

In this paper, through the analysis of the characteristics of mechanical parts surface defects proposed mining based on target feature detection method for defect detection; the general features of mechanical parts surface defects, determine the threshold in image segmentation, in order to achieve the target defects segmentation; on this basis, to achieve accurate detection of surface defects of mechanical parts; because the algorithm is simple and reduces the amount of calculation and is easy to realize the hardware to online fast automatic detection provides a way.

\section{Acknowledgements}

This work was supported by College student's innovation and Entrepreneurship Program Funded (Program No. 1578).

\section{Reference}

[1] WU Pingchuan, LU Tongjun, WANG Yan. Machine vision technology and nondestructive of the surface defects in strip steel[J]. Nondestructive Testing, 2000, 22(1): 3-16

[2] LIU Zhong, WU Jie, ZHANG Hua. Design and practice of the hot strip surface quality inspection system[J]. Baosteel Technology, 2005,13(6): 57-60

[3] Zhong Cai. The application of edge detection algorithm in image preprocessing [J]. Software,2013,34 (1): 158-159

[4] Xie Peijun. A genetic algorithm based on membrane computing image segmentation method [J]. Software,2014, 35(9): 80-85

[5] Guohui Li, Jingfang Shi, Hongsen Luo and Miangang Tang. A computational model of vision attention for inspection of surface quality in production line[J]. Machine Vision and Applications, 2013, 24:835-844

[6] Chen Cheng, Chen Yao, Chen Xiaojie, et al. Design and implementation of an online detection system for steel strip surface defects [J].Software,2013,34 (11): 18-20

[7] Chen Hanxin, Liu Cen, Yang Shiqi. A method for the detection and diagnosis of gear crack fault [J]. Journal of Wuhan Institute of Technology,2014，36(9): 53-58

[8] Guan shengqi, Shi Hongyu, Wang Yanni, [J]. research on iron and steel strip defect detection based on image zero mean.

[9] Yang Jianguo, Xiao Rong, Li Beizhi, Cui Zhexue, Zhou Hu. Tool wear detection technology based on machine vision [J]. Journal of Donghua University (NATURAL SCIENCE EDITION),2012, 38(5):505-508

[10] Guan shengqi, Qu Yunxian, yuan. According to wavelet homomorphic filtering tool wear detection of [J]. mechanical science and technology,2013，32（11): 1703-1707 